\title{
Enriched environment contributes to recovery of visual acuity and increases perineuronal nets in monocular-deprived animals
}

\author{
Nonata Trévia, Izabela N. F. Almeida, Poliana S. Oliveira, Laura V. Warwick, Viviane Marques, \\ Denise C. dos Santos, Dario C. Paulo, Maria L. Viegas and Cristovam W. Picanço-Diniz \\ Universidade Federal do Pará, Belém, PA, Brazil
}

\begin{abstract}
The aim of the present study was to analyze the influence of enriched environment on the distribution of perineuronal nets (PNNs) using a stereogically based unbiased protocol and visual acuity in adult Swiss albino mice that underwent monocular deprivation during the critical period of postnatal development. Eight female Swiss albino mice were monocular deprived on postnatal day 10 and divided into two groups at weaning: standard environment (SE group, $n=4)$ and enriched environment (EE group, $n=$ 4). After 3 months, all of the mice were subjected to grating visual acuity tests, sacrificed, and perfused with aldehyde fixative. The brains were removed and cut at $70 \mu \mathrm{m}$ thickness in a vibratome and processed for lectin histochemical staining with Wisteria floribunda agglutinin (WFA). Architectonic limits of area 17 were conspicuously defined by WFA histochemical staining, and the optical fractionator stereological method was applied to estimate the total number of PNNs in the supragranular, granular, and infragranular layers. All groups were compared using Student's $t$-test at a 95\% confidence level. Comparative analysis of the average PNN estimations revealed that the EE group had higher PNNs in the supragranular layer (2726.33 \pm 405.416 , mean \pm standard deviation) compared with the SE group (1543.535 \pm 260.686 ; Student's $t$-test, $p=.0495)$. No differences were found in the other layers. Visual acuity was significantly lower in the SE group ( 0.55 cycles/degree) than in the EE group (1.06 cycles/ degree). Our results suggest that the integrity of the specialized extracellular matrix PNNs of the supragranular layer may be essential for normal visual acuity development. Keywords: monocular deprivation, critical period, visual cortex, perineuronal nets, visual acuity, stereology.
\end{abstract}

Received 13 January 2011; received in revised form 22 February 2011; accepted 30 March 2011. Available on line 15 june 2011

\section{Introduction}

Critical periods are associated with experiencedependent plasticity in various species and sensory systems and are related to an extreme form of more generalized sensitivity, in which the nervous system is able to respond and modulate its activity and connections based on natural experience, particularly during the postnatal period (Hensch, 2004). The postnatal critical period window has been most extensively studied with regard to the visual system using classic experiments that typically comprise manipulations of sensory inputs, such as monocular deprivation in rodents, mammals,

Nonata Trévia, Izabela N. F. Almeida, Poliana S. Oliveira, Laura V. Warwick, Viviane Marques, Denise C. dos Santos, Dario C. Paulo, Maria L. Viegas, Cristovam W. Picanço-Diniz, Laboratório de Investigações em Neurodegeneração e Infecção, Hospital Universitário João de Barros Barreto, Universidade Federal do Pará, Brazil. Correspondence regarding this article should be directed to: Nonata Trévia, Universidade Federal do Pará, Hospital Universitário João de Barros Barreto, Laboratório de Investigações em Neurodegeneração e Infecção. Rua dos Mundurucus, 4487, Guamá, Belém, Pará, 66073-000 Brasil.E-mail: ntrevia@yahoo.com.br and primates (Berardi, Pizzorusso, \& Maffei, 2000). The pioneering experiments of Wiesel and Hubel demonstrated that the two eyes interact and compete for cortical territory, thereby rewiring visual connections (Wiesel \& Hubel, 1963).

This manipulation of the visual experience results in an imbalance of inputs between the two eyes, and a shift of ocular dominance occurs in favor of the opened eye. Consequently, the deprived eye experiences a loss of visual acuity, termed amblyopia. In adulthood, after the closure of the critical period and consequent reduction of cortical plasticity, achieving modifications in cortical circuitry and recovery of visual deficits induced by amblyopia is difficult.

On the other hand, an enriched environment (EE), previously defined as "a combination of complex inanimate and social stimulation," (Sztainberg \& Chen, 2010, p. 1535) is responsible for various brain changes that can enhance plasticity and increase dendritic arborization, gliogenesis, neurogenesis, and learning and memory in the adult brain (van Praag, Kempermann, \& Gage, 2000). In an EE, animals are maintained in large cages with numerous other animals and a complex environment that provides them the 
opportunity to increase exploratory behavior, sensorymotor stimulation, and complex social interactions.

Critical period time-windows are regulated by a cascade of molecular determinants, including neurotrophins, variations in $N$-methyl-D-aspartate receptor subunit composition and $\gamma$-aminobutyric acid inhibitory circuitry, and the maturation of perineuronal nets (PNNs) (Berardi et al., 2000). Recent studies have suggested that the maturation of inhibitory circuits is a key determinant in the initiation and termination of plasticity windows. Furthermore, the complete development of PNNs appears to coincide with the end of the critical period when cortical plasticity decreases (Pizzorusso et al., 2002).

The present study tested whether environmental enrichment in adulthood recovers impairments in visual acuity induced by monocular deprivation early in life and whether environmental enrichment changes the distribution of PNNs in the laminar organization of the primary visual cortex in rodents.

\section{Methods}

\section{Animals and monocular deprivation}

All procedures were performed according to the "Principles of Laboratory Animal Care" and approved by the ethics committee of the Federal University of Pará. Pregnant Swiss albino mice were donated by Instituto Evandro Chagas and transported to the animal house of the Infection and Neurodegeneration Laboratory. After birth, on postnatal day 10 , female pups received general anesthesia (2,2,2-tribromoethanol, $0.8 \mathrm{ml} / 5 \mathrm{~g}$ body weight, i.p.) and had their eye-lid sutured with 6-0 nylon silk. After surgery, a topical antibacterial ophthalmic agent (3.5 mg neomycin and $0.1 \%$ dexamethazone) was applied to the eye once per day for 3 days. The eye-lid sutures were checked daily. If an animal presented a minimum aperture in the suture, then it was anesthetized and sacrificed.

After weaning, on postnatal day 21, the animals were divided into two experimental groups: standard environment (SE group, $n=4$ ) and enriched environment (EE group, $n=4)$. Animals in the SE group were kept in white plastic cages $(12.3 \times 17.3 \times 27.7 \mathrm{~cm})$, with two animals per cage. Animals in the EE group were kept in groups of four animals in large plastic cages $(16 \mathrm{x}$ $31.1 \times 46 \mathrm{~cm}$ ) with a variety of colored toys, running wheels, ladders, ropes, and tunnels to stimulate, visual, somatosensory, motor, and exploratory activity.

All animals were kept in a room maintained at $23 \pm$ $2^{\circ} \mathrm{C}$ under a $12 \mathrm{~h} / 12 \mathrm{~h}$ light/dark cycle (lights on at 7:00 AM) and had ad libitum access to water and food until the end of the experiment.

\section{Visual water-box test}

After 3 months under enriched or standard environments, visual acuity was assessed in the visual water-box apparatus.
As previously described (Prusky, West, \& Douglas, 2000), the water box was $140 \mathrm{~cm}$ long x $80 \mathrm{~cm}$ wide and had $55 \mathrm{~cm}$ high walls. The pool was wider at one end $(80 \mathrm{~cm})$ than the other $(25 \mathrm{~cm}$ ) and painted gray. A midline divider (40 cm high x 27 $\mathrm{cm}$ long) was placed into the pool, bisecting it along its long axis. The length of the divider sets the choice point and defines the angular value to estimate the spatial frequency value (Prusky et al., 2000). A grating and non-patterned cardboard with equal luminance values were randomly displayed at the wide end of the trapezoidal-shaped tank. The pool was filled with non-toxic bluish-gray water to a depth of $15 \mathrm{~cm}$. The animals were trained to swim toward the larger end of the water-box and at a fixed distance choose the wall that displayed the grating and escape to a submerged platform hidden below it. The mice were considered to accomplish the task when $75 \%$ correct choices occurred in four consecutive trials. Different spatial frequencies $(0.03,0.06,0.12,0.24$, and 0.45 cycles/ degree) with fixed luminance $\left(0.093 \mathrm{~cd} / \mathrm{m}^{2}\right)$ were used to determine visual acuity. All spatial frequencies (0.03-0.45 cycles/degree) were tested for a total of 10 consecutive days. When the animal reached $75 \%$ correct choices, the visual cue was changed to a higher frequency. If, after 3 training days, an animal did not reach $75 \%$ correct choices for a specific spatial frequency, then the task was ended, and the previously tested spatial frequency was considered the final discriminative value for that animal.

\section{Histochemical procedures}

After the visual task, all animals were deeply anesthetized with 2,2,2 tribromoethanol at $0.8 \mathrm{ml} / 5$ g of body weight, i.p., and transcardially perfused with heparinized saline for $10 \mathrm{~min}$, followed by $4 \%$ paraformaldehyde in $0.1 \mathrm{M}$ phosphate buffer, $\mathrm{pH}$ 7.27.4 , for $30 \mathrm{~min}$. After perfusion and craniotomy, the brains were left overnight in $2 \%$ paraformaldehyde at $4^{\circ} \mathrm{C}$ for post-fixation. The hemispheres were then sectioned on a vibratome at $70 \mu \mathrm{m}$ thickness. One of every five coronal sections was collected to stain PNNs using a Wisteria floribunda histochemical reaction. After rinsing in TRIS-triton 5\% buffer for 20 min, the sections were incubated for approximately 24 $\mathrm{h}$ in a solution that contained $20 \mu \mathrm{g} / \mathrm{ml}$ biotinylated Wisteria floribunda agglutinin (WFA, Vector Labs, Burlingame, CA, USA) in 5\% Triton X-100 TRIS buffer. At the end of the incubation period, the sections were rinsed in TRIS-buffered saline (TBS), placed in $\mathrm{ABC}$ solution for $1 \mathrm{~h}$, washed again, and reacted to reveal PNNs using a glucose oxidasediaminobenzidine-nickel protocol (Shu, Ju, \& Fan, 1988). All steps were performed with gentle and continuous agitation at $4^{\circ} \mathrm{C}$. After a final rinse with phosphate-buffered saline, the sections were mounted, dehydrated, cleared, and sealed with Entellan (Merck, NJ, USA). All chemicals used in this study were supplied by Sigma (Poole, UK) or Vector Labs. 


\section{Stereological design}

The limits of V1 were defined by clear architectonic differences in the neuropil, in which V1 appears darker than adjacent areas (Fig. 2 top) Wisteria floribunda histochemistry selectively labels the $N$-acetylgalactosamine $\beta 1$ residues of glycoproteins (Murakami et al., 1999), revealing PNNs within the extracellular matrix and conspicuously defining the architectonic limits and layers in the neocortex or hippocampal regions (Alpár, Gärtner, Härtig, \& Brückner, 2006; Bruckner, Grosche, Hartlage-Rubsamen, Schmidt, \& Schachner, 2003). The PNN counting procedure included two types of nets: type I (corresponding to perisomatic and faint primary dendrites) and type II (corresponding to perisomatic and peridendritic nets, including distinct secondary and tertiary branches).

At all levels in the histological sections, we delineated the granular, supragranular, and infragranular layers of V1, digitizing directly from sections with a low resolution (3.2x objective) on an Optiphot- 2 microscope (Nikon, Tokyo, Japan) equipped with a motorized stage (MAC200, Ludl Electronic Products, Hawthorne, NY, USA). This system was coupled to a computer that ran Stereoinvestigator software (MicroBrightField, Williston, VT, USA) and used to store and analyze the $x, y$, and $z$ coordinates of the digitized points. To detect and count the objects of interest unambiguously with the dissector probe, the low-resolution objective was replaced with a high-resolution 60x oil immersion planapochromatic objective (NIKON, NA 1.4). Thus, all stereological estimations began with the delineation of the region of interest in horizontal sections, where the layers and limits of V1 were unambiguously identified and outlined. At each counting site, the thickness of the section was carefully assessed with the high-resolution objective, and the fine focus of the microscope was used to define the top and bottom of the section in each counting site. Because both the thickness and distribution of the cells in the section varied, the total number of objects of interest was weighted with the section thickness. All objects that came into focus inside the counting frame were counted and added to the total number of markers, provided that they were entirely within the counting frame or intersected the acceptance line without touching the rejection line (Gundersen \& Jensen, 1987). The counting boxes were randomly and systematically placed within a grid.

Tables 1-3 present the experimental parameters and average counting results obtained with the optical fractionator. Using this optical fractionation of sections, we were able to determine the V1 number of markers $\left(\Sigma Q^{-}\right)$in adult female Swiss albino mice. The grid sizes were adapted to achieve an acceptable coefficient of error (CE). For the $\mathrm{CE}$ of the total cell counts for each subject in the present study, we adopted the one-stage systematic sampling procedure (Scheaffer $\mathrm{CE}$ ) used previously and validated elsewhere (Glaser \& Wilson 1998). The level of acceptable errors for stereological estimations was defined as the ratio between the intrinsic error introduced by the methodology and the coefficient of variation (Glaser \& Wilson, 1998; Slomianka \& West, 2005). The CE expresses the accuracy of the cell number estimates, and a value of $\mathrm{CE} \leq 0.05$ was deemed appropriate for the present study because variance introduced by the estimation procedure contributed little to the observed group variance (Glaser \& Wilson, 1998; Slomianka \& West, 2005) The experimental parameters were established in pilot experiments and uniformly applied to all animals. Eight female Swiss albino mice had their eye-lid sutured on postnatal day 10 and were then divided into two experimental groups: standard environment (SE group; $n=4$ ) and enriched

Table 1. Stereological parameters and total number of perineuronal nets in the supragranular layer of the primary visual cortex in female Swiss albino mice raised in a standard environment or enriched environment.

\begin{tabular}{ccccccccc}
\hline Animal & $\begin{array}{c}\text { Counting } \\
\text { frame area } \\
\text { (a) }\left(\mu \mathrm{m}^{2}\right)\end{array}$ & $\begin{array}{c}\text { Sampling } \\
\text { grid area } \\
(\mathrm{A})(x, y) \\
\left(\mu \mathrm{m}^{2}\right)\end{array}$ & asf & Tsf & ssf & $\begin{array}{c}\text { No. of } \\
\text { sampling } \\
\text { sites }\end{array}$ & $\begin{array}{c}\text { No. of } \\
\text { sections }\end{array}$ & SQ \\
\hline SE 2 & 3600 & 8100 & 0.44 & 0.60 & $1 / 5$ & 282 & 10 & 82 \\
SE 3 & 3600 & 8100 & 0.44 & 0.60 & $1 / 5$ & 217 & 7 & 91 \\
SE 5 & 3600 & 8100 & 0.44 & 0.63 & $1 / 5$ & 162 & 7 & 48 \\
SE 6 & 3600 & 8100 & 0.44 & 0.58 & $1 / 5$ & 253 & 10 & 109 \\
EE 1 & 3600 & 8100 & 0.44 & 0.55 & $1 / 5$ & 274 & 8 & 155 \\
EE 2 & 3600 & 8100 & 0.44 & 0.48 & $1 / 5$ & 303 & 10 & 153 \\
EE 6 & 3600 & 8100 & 0.44 & 0.60 & $1 / 5$ & 282 & 8 & 92 \\
EE 9 & 3600 & 8100 & 0.44 & 0.51 & $1 / 5$ & 211 & 7 & 112 \\
\hline
\end{tabular}

asf, area sampling fraction $=\mathrm{a}($ frame area) $/ \mathrm{A}$ (grid area); tsf, thickness sampling fraction $=$ dissector height/number weighted mean section thickness; ssf, section sampling fraction; SQ, total markers counted. 
Table 2. Stereological parameters and total number of perineuronal nets in the granular layer of the primary visual cortex in female Swiss albino mice raised in a standard environment or enriched environment.

\begin{tabular}{|c|c|c|c|c|c|c|c|c|}
\hline Animal & $\begin{array}{l}\text { Counting } \\
\text { frame area } \\
\text { (a) }\left(\mu \mathrm{m}^{2}\right)\end{array}$ & $\begin{array}{c}\text { Sampling } \\
\text { grid area } \\
(\mathrm{A})(x, y) \\
\left(\mu \mathrm{m}^{2}\right)\end{array}$ & asf & tsf & ssf & $\begin{array}{c}\text { No. of } \\
\text { sampling } \\
\text { sites }\end{array}$ & $\begin{array}{l}\text { No. of sec- } \\
\text { tions }\end{array}$ & SQ \\
\hline SE 2 & 3600 & 8100 & 0.44 & 0.61 & $1 / 5$ & 262 & 9 & 178 \\
\hline SE 3 & 3600 & 8100 & 0.44 & 0.59 & $1 / 5$ & 268 & 8 & 219 \\
\hline SE 5 & 3600 & 8100 & 0.44 & 0.60 & $1 / 5$ & 161 & 6 & 91 \\
\hline SE 6 & 3600 & 8100 & 0.44 & 0.59 & $1 / 5$ & 226 & 9 & 134 \\
\hline EE 1 & 3600 & 8100 & 0.44 & 0.51 & $1 / 5$ & 251 & 9 & 176 \\
\hline EE 2 & 3600 & 8100 & 0.44 & 0.45 & $1 / 5$ & 292 & 10 & 197 \\
\hline EE 6 & 3600 & 8100 & 0.44 & 0.57 & $1 / 5$ & 336 & 8 & 183 \\
\hline EE 9 & 3600 & 8100 & 0.44 & 0.52 & $1 / 5$ & 192 & 7 & 166 \\
\hline
\end{tabular}

asf, area sampling fraction $=\mathrm{a}($ frame area) $/ \mathrm{A}$ (grid area); tsf, thickness sampling fraction $=$ dissector height/number weighted mean section thickness; ssf, section sampling fraction; SQ, total markers counted.

Table 3. Stereological parameters and total number of perineuronal nets in the infragranular layer of the primary visual cortex in female Swiss albino mice raised in a standard environment or enriched environment.

\begin{tabular}{|c|c|c|c|c|c|c|c|c|}
\hline Animal & $\begin{array}{l}\text { Counting } \\
\text { frame area } \\
\text { (a) }\left(\mu \mathrm{m}^{2}\right)\end{array}$ & $\begin{array}{c}\text { Sampling } \\
\text { grid area } \\
(\mathrm{A})(x, y) \\
\left(\mu \mathrm{m}^{2}\right)\end{array}$ & asf & tsf & ssf & $\begin{array}{c}\text { No. of } \\
\text { sampling } \\
\text { sites }\end{array}$ & $\begin{array}{l}\text { No. of sec- } \\
\quad \text { tions }\end{array}$ & SQ \\
\hline SE 2 & 3600 & 8100 & 0.44 & 0.62 & $1 / 5$ & 298 & 10 & 213 \\
\hline SE 3 & 3600 & 8100 & 0.44 & 0.62 & $1 / 5$ & 253 & 7 & 204 \\
\hline SE 5 & 3600 & 8100 & 0.44 & 0.60 & $1 / 5$ & 213 & 6 & 118 \\
\hline SE 6 & 3600 & 8100 & 0.44 & 0.60 & $1 / 5$ & 288 & 9 & 175 \\
\hline EE 1 & 3600 & 8100 & 0.44 & 0.56 & $1 / 5$ & 309 & 8 & 232 \\
\hline EE 2 & 3600 & 8100 & 0.44 & 0.48 & $1 / 5$ & 371 & 10 & 246 \\
\hline EE 6 & 3600 & 8100 & 0.44 & 0.59 & $1 / 5$ & 363 & 8 & 167 \\
\hline EE 9 & 3600 & 8100 & 0.44 & 0.55 & $1 / 5$ & 229 & 7 & 179 \\
\hline
\end{tabular}

asf, area sampling fraction $=\mathrm{a}($ frame area) $/ \mathrm{A}$ (grid area); tsf, thickness sampling fraction $=$ dissector height/number weighted mean section thickness; ssf, section sampling fraction; SQ, total markers counted.

environment (EE group; $n=4)$. Enriched environmental conditions consisted of plastic cages $(32 \times 39 \times 16.5$ $\mathrm{cm})$ with chopped rice straw bedding equipped with rod bridges, tunnels, running wheels, and toys made of plastic, wood, or metal with different forms and colors that were changed every week. The SE cages corresponded to plastic cages with the same dimensions and chopped rice straw bedding but without equipment or toys. Each cage housed 12-15 mice. All mice had free access to water and food, with a $12 \mathrm{~h} / 12 \mathrm{~h}$ light/dark cycle. The tests occurred during the light cycle.

All procedures were performed according to the "Principles of Laboratory Animal Care" and approved by the ethics committee of the Federal University of Pará.

\section{Photomicrographic documentation and processing}

To obtain digital photomicrographs, we used a digital camera (Microfire, Optronics, CA, USA) coupled to an Nikon Optiphot-2 microscope (Nikon, Tokyo, Japan). Digital photomicrographs were processed using Adobe Photoshop 7.0.1 CS2 software (San Jose, CA, USA) for scaling and adjusting the levels of brightness and contrast. For the figures, selected pictures were taken of sections from the animals in each experimental group with the total number of objects of interest nearest the mean value of each region of interest.

\section{Results}

We measured visual acuity in 3-month-old monoculardeprived animals using the visual water-box test. Animals raised in the SE had a visual acuity cut-off value of 0.55 cycles/degree. Animals raised in the EE had a visual acuity cut-off value of 1.06 cycles/degree (Fig. 1).

To determine whether PNN maturation is associated with improved performance in the visual acuity test, 


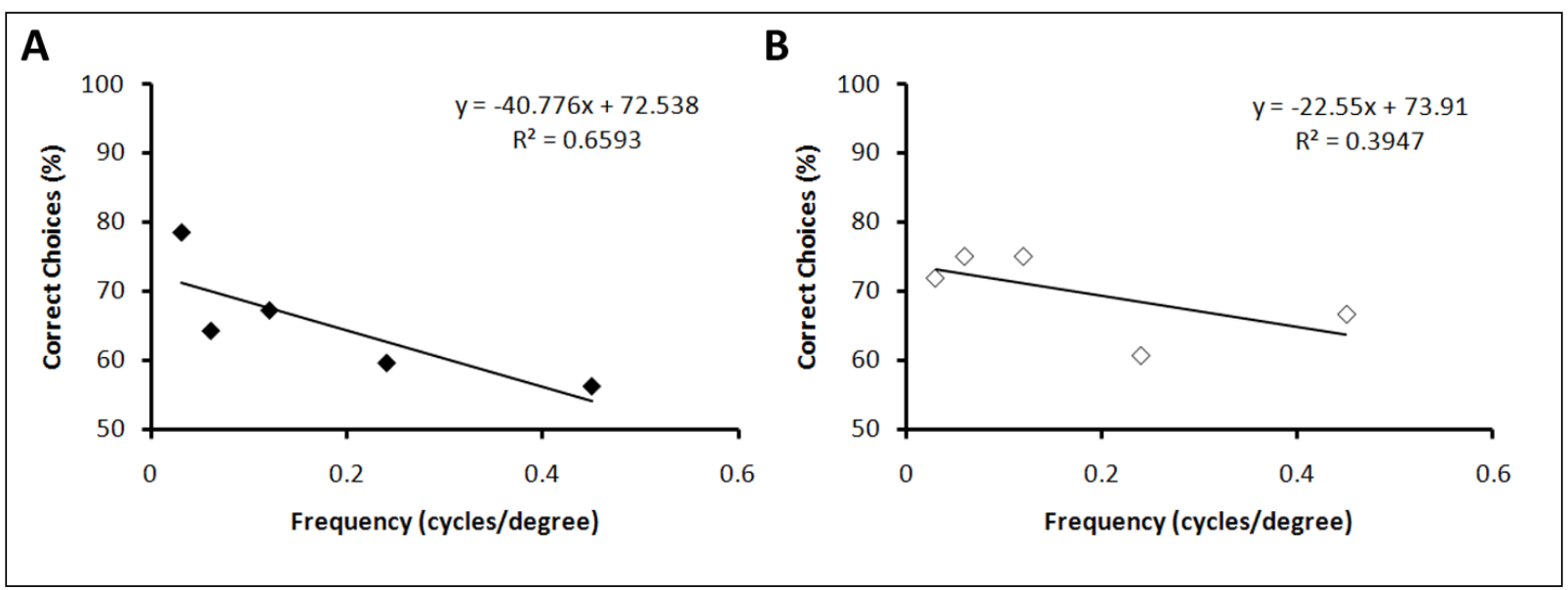

Figure 1. Relationship between correct choices (\%) and spatial frequency (cycles/degree) in 3-month-old monocular-deprived female Swiss albino mice. Visual acuity is expressed as the spatial frequency that corresponded to $50 \%$ correct choices on the linear regression function. A: Standard environment (SE). B: Enriched environment (EE).

we quantified the number of PNNs stained by Wisteria floribunda lectin in the contralateral primary visual cortex using stereological procedures. Tables 4 and 5 present the optical fractionator estimations of PNNs in V1 of each subject and the mean values for the experimental groups. Laminar quantification of the PNNs in the supragranular layers of the visual cortex that comprise layers 1,2 , and
3 revealed that the EE group had a significantly higher number of PNNs $(2726.33 \pm 405.416$, mean \pm standard error) than the SE group $(1543.535 \pm 260.686$, mean \pm standard error; Student's $t$-test, $p=.0495$; Fig. 2). To assure that differences between animal groups were attributable only to the experimental manipulations and that biological variation was responsible for more than
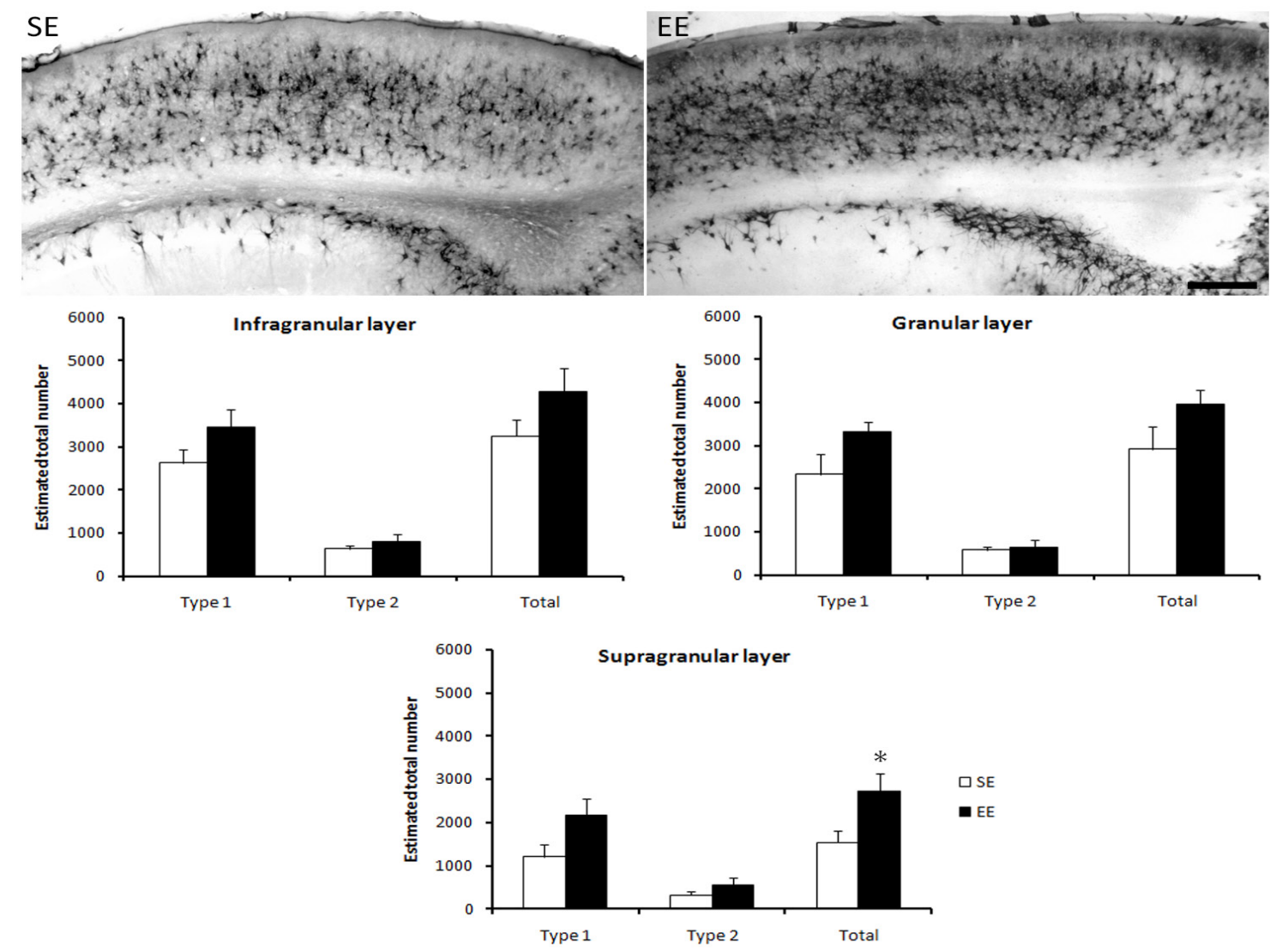

Figure 2. (Top) Photomicrographs of contralateral primary visual cortices of mice raised in either a standard environment (SE) or enriched environment (EE). Scale bar $=250 \mu \mathrm{m}$. (Bottom) Stereological quantification of perineuronal nets, revealed by Wisteria floribunda lectin in the infragranular, granular, and supragranular layers of the contralateral primary visual cortex. Errors bars indicate SEM. * $p=.0495$. 
$50 \%$ of the total variation, we determined the coefficient of biological variation (CBV) as a percentage of the coefficient of variation (Tables 4 and 5). Nonsignificant differences were detected in the granular and infragranular layers of the contralateral primary visual cortex.

\section{Discussion}

Altogether, the present results suggest that early and prolonged exposure to an EE may contribute to partial recovery from visual impairments induced by monocular deprivation. Furthermore, these results may be associated with an increase in the total number of PNNs in the primary visual cortex. Our behavioral data confirmed that Swiss albino mice were an appropriate model for inducing visual discrimination deficits by monocular deprivation during the critical period, which reduced visual acuity. Thus, the present results are consistent with previous reports that showed that EE promoted the recovery of visual

Table 4. Total number of perineuronal nets in each layer of the contralateral primary visual cortex in female Swiss albino mice raised in a standard environment.

\begin{tabular}{|c|c|c|c|c|c|c|}
\hline \multirow{2}{*}{ Animal } & \multicolumn{2}{|c|}{ Supragranular } & \multicolumn{2}{|c|}{ Granular } & \multicolumn{2}{|c|}{ Infragranular } \\
\hline & $\mathrm{N}$ & $\mathrm{CE}$ & $\mathrm{N}$ & $\mathrm{CE}$ & $\mathrm{N}$ & $\mathrm{CE}$ \\
\hline SE 2 & 1528.08 & 0.09 & 3252.62 & 0.07 & 3834.45 & 0.06 \\
\hline SE 3 & 1691.11 & 0.09 & 4163.16 & 0.06 & 3672.31 & 0.06 \\
\hline SE 5 & 851.08 & 0.13 & 1694.2 & 0.09 & 2197.98 & 0.08 \\
\hline SE 6 & 2103.87 & 0.08 & 2547.42 & 0.07 & 3286.7 & 0.06 \\
\hline Mean & 1543.53 & 0.097 & 2914.35 & 0.07 & 3247.86 & 0.06 \\
\hline S.D. & 521.37 & 0.022 & 1048.38 & 0.012 & 736.65 & 0.010 \\
\hline $\mathrm{CV}^{2}=(\mathrm{S} \cdot \mathrm{D} / \text { Mean })^{2}$ & 0.1140 & & 0.1294 & & 0.0514 & \\
\hline $\mathrm{CE}^{2}$ & & 0.009 & & 0.0049 & & 0.0036 \\
\hline $\mathrm{CE}^{2} / \mathrm{CV}^{2}$ & 0.0789 & & 0.0378 & & 0.0700 & \\
\hline $\mathrm{CVB}^{2}=\left(\mathrm{CV}^{2}-\mathrm{CE}^{2}\right)$ & 0.105 & & 0.1245 & & 0.0478 & \\
\hline $\mathrm{CVB}^{2}\left(\%\right.$ of $\left.\mathrm{CV}^{2}\right)$ & $92.1 \%$ & & $96.21 \%$ & & $92.99 \%$ & \\
\hline
\end{tabular}

$\mathrm{CVB}^{2}=\mathrm{CV}^{2}-\mathrm{CE}^{2}\left(\mathrm{CV}\right.$, coefficient of variation; $\mathrm{CE}$, estimated $\mathrm{CE}$ [Scheaffer]; $\mathrm{CVB}^{2}$, coefficient of biological variation). $\mathrm{N}$, estimated total number of PNNs using optical fractionator method; S.D., standard deviation.

Table 5. Total number of perineuronal nets for each layer of the contralateral primary visual cortex in female Swiss albino mice raised in an enriched environment.

\begin{tabular}{|c|c|c|c|c|c|c|}
\hline \multirow{2}{*}{ Animal } & \multicolumn{2}{|c|}{ Supragranular } & \multicolumn{2}{|c|}{ Granular } & \multicolumn{2}{|c|}{ Infragranular } \\
\hline & $\mathrm{N}$ & $\mathrm{CE}$ & $\mathrm{N}$ & $\mathrm{CE}$ & $\mathrm{N}$ & $\mathrm{CE}$ \\
\hline EE 1 & 3121.55 & 0.07 & 3859.65 & 0.07 & 4664.21 & 0.06 \\
\hline EE 2 & 3592.56 & 0.08 & 4896.48 & 0.06 & 5647.43 & 0.06 \\
\hline EE 6 & 1728.07 & 0.10 & 3544.19 & 0.07 & 3157.39 & 0.07 \\
\hline EE 9 & 2463.14 & 0.08 & 3557.69 & 0.07 & 3611.23 & 0.06 \\
\hline Mean & 2627.33 & 0.08 & 3964.50 & 0.06 & 4270.06 & 0.06 \\
\hline S.D. & 810.83 & 0.01 & 638.15 & 0.005 & 1114.24 & 0.005 \\
\hline $\mathrm{CV}^{2}=(\mathrm{S} . \mathrm{D} / \text { Mean })^{2}$ & 0.0952 & & 0.0259 & & 0.0680 & \\
\hline $\mathrm{CE}^{2}$ & & 0.0064 & & 0.0036 & & 0.0036 \\
\hline $\mathrm{CE}^{2} / \mathrm{CV}^{2}$ & 0.0672 & & 0.1389 & & 0.0529 & \\
\hline $\mathrm{CVB}^{2}=\left(\mathrm{CV}^{2}-\mathrm{CE}^{2}\right)$ & 0.0888 & & 0.0223 & & 0.0644 & \\
\hline $\mathrm{CVB}^{2}\left(\%\right.$ of $\left.\mathrm{CV}^{2}\right)$ & $93.27 \%$ & & $86.10 \%$ & & $94.70 \%$ & \\
\hline
\end{tabular}

$\mathrm{CVB}^{2}=\mathrm{CV}^{2}-\mathrm{CE}^{2}\left(\mathrm{CV}\right.$, coefficient of variation; $\mathrm{CE}$, estimated $\mathrm{CE}$ [Scheaffer]; $\mathrm{CVB}^{2}$, coefficient of biological variation). $\mathrm{N}$, estimated total number of PNNs using the optical fractionator method; S.D., standard deviation. 
acuity in adult monocular-deprived animals but not at the expense of PNN damage.

Although a few reports quantified PNN changes that correlated with visual acuity and an EE, their quantitative approach was not based on unbiased stereological estimations (Hilbig, Bidmon, Steingruber, Reinke, \& Dinse, 2002; Pizzorusso et al., 2002; Ciucci et al., 2007; Sale et al., 2007). Consequently, quantitative associations between PNNs and changes in visual acuity in murine models and the effect of an EE have previously not been subjected to stereological analysis. The present report estimated the total number of PNNs using an optical fractionator method, which is an accurate quantification method that combines the properties of an optical dissector and fractionator and has been used in numerous studies to determine cell numbers in multiple brain regions (West, 2002). The optical fractionator method is unaffected by histological changes, shrinkage, or damageinduced expansion by injury, an issue that is particularly important when studying brain-induced changes (West, Slomianka, \& Gundersen, 1991).

Perineuronal nets reach their mature pattern late in the postnatal development period, coinciding with the end of the critical period, contributing to the stabilization of synaptic connections, and limiting plasticity (Pizzorusso et al., 2002). In monoculardeprived animals, the reactivity to floribunda agglutinin is reduced in adulthood, suggesting that the lack of visual stimulation in the contralateral cortex delays the maturation of the extracellular matrix. However, in the present study, monocular deprived animals raised in an EE exhibited increases in PNN laminar distribution and visual acuity, which may be related to the formation and stabilization of new synapses that were induced by the intense visual stimulation experienced in the EE. Consistent with the present results, in aging animals, the senescent loss of PNNs in the somatosensory cortex is prevented by enriched environmental conditions that are able to promote new functional plasticity (Hilbig et al., 2002). Additionally, PNNs in the adult brain appear to be essential for the maintenance of long-term potentiation in Schaffer collateral cells in the CA1 region, which modulates several forms of synaptic plasticity (Bukalo, Schachner, \& Dityatev, 2001). Visual experience, such as in an EE, induces long-term potentiation in the primary visual cortex, and PNNs are essential for longterm potentiation (Cooke \& Bear, 2010).

The grating acuity of normal and monoculardeprived mice using the visual water task was previously reported to be equivalent to normal mice (approximately 0.5 cycles/degree; Prusky \& Douglas, 2003). In the present study, we obtained similar results in monoculardeprived subjects raised in similar conditions (SE group: visual acuity $=0.55$ cycles/degree), whereas animals in the EE group achieved 1.06 cycles/degree. We confirmed that visual acuity recovered in the EE group (Sale et al., 2007) and suggest that this recovery may be related to the formation and stabilization of new synapses that are protected by the formation of new PNNs. This new formation and protection may be induced by the more intense visual stimulation present in the EE. The results confirmed that an EE may be a noninvasive therapeutic method that could contribute to the recovery of cortical function after pathological changes, which may be applied to other neurological diseases.

\section{Acknowledgments}

This work was funded by the Brazilian Research Council (CNPq). CWPD was funded by FINEP-IBNnet.

\section{References}

Alpár, A., Gärtner, U., Härtig, W., \& Brückner, G. (2006). Distribution of pyramidal cells associated with perineuronal nets in the neocortex of rat. Brain Research, 1120(1), 13-22.

Berardi, N., Pizzorusso, T., \& Maffei, L. (2000). Critical periods during sensory development. Current Opinion in Neurobiology, 10(1), 138-145.

Bruckner, G., Grosche, J., Hartlage-Rubsamen, M., Schmidt, S., \& Schachner, M. (2003). Region and lamina-specific distribution of extracellular matrix proteoglycans, hyaluronan and tenascin-R in the mouse hippocampal formation. Journal of Chemical Neuroanatomy, 26, 37-50.

Bukalo, O., Schachner, M., \& Dityatev, A. (2001). Modification of extracellular matrix by enzymatic removal of chondroitin sulfate and by lack of tenascin-R differentially affects several forms of synaptic plasticity in the hippocampus. Neuroscience, 104, 359369.

Ciucci, F., Putignano, E., Baroncelli, L., Landi, S., Berardi, N., \& Maffei, L. (2007). Insulin-like growth factor 1 (IGF-1) mediates the effects of enriched environment (EE) on visual cortical development. PLoS One, 2(5), e475.

Cooke, S.F., \& Bear, M.F. (2010). Visual experience induces long-term potentiation in the primary visual cortex. Journal of Neuroscience, 30, 16304-16313.

Glaser, E. M., \& Wilson, P.D. (1998). The coefficient of error of optical fractionator population size estimates: a computer simulation comparing three estimators. Journal of Microscopy, $192(\mathrm{Pt} \mathrm{2})$, $163-171$

Gundersen, H. J., \& Jensen, E.B. (1987). The efficiency of systematic sampling in stereology and its prediction. Journal of Microscopy, 147 (Pt 3), 229-263.

Hensch, T.K. (2004). Critical period regulation. Annual Review of Neuroscience, 27, 549-579.

Hilbig, H., Bidmon, H.J., Steingruber, S., Reinke, H., \& Dinse, H.R. (2002). Enriched environmental conditions reverse agedependent gliosis and losses of neurofilaments and extracellular matrix components but do not alter lipofuscin accumulation in the hindlimb area of the aging rat brain. Journal of Chemical Neuroanatomy, 23, 199-209.

Murakami, T., Ohtsuka, A., Su, W.D., Taguchi, T., Oohashi, T., Murakami, T., Abe, K., \& Ninomiya, Y., (1999). The extracellular matrix in the mouse brain: its reactions to endo-alpha-Nacetylgalactosaminidase and certain other enzymes. Archives of Histology and Cytology, 62, 273-281.

Pizzorusso, T., Medini, P., Berardi, N., Chierzi, S., Fawcett, J.W., \& Maffei, L. (2002). Reactivation of ocular dominance plasticity in the adult visual cortex. Science, 298, 1248-1251.

Prusky, G.T., West, P.W., \& Douglas, R.M. (2000). Behavioral assessment of visual acuity in mice and rats. Vision Research, 40, 2201-2209.

Prusky, G.T., \& Douglas, R.M. (2003). Developmental plasticity of mouse visual acuity. European Journal of Neuroscience, 17(1), 167-173.

Sale, A., Maya Vetencourt, J.F., Medini, P., Cenni, M.C., Baroncelli, L., De Pasquale, R., \& Maffei, L. (2007). Environmental enrichment 
in adulthood promotes amblyopia recovery through a reduction of intracortical inhibition. Nature Neuroscience, 10, 679-681.

Shu, S.Y., Ju, G., \& Fan, L.Z. (1988). The glucose oxidase-DABnickel method in peroxidase histochemistry of the nervous system. Neuroscience Letters, 85, 169-171.

Slomianka, L., \& West, M. J. (2005). Estimators of the precision of stereological estimates: an example based on the CA1 pyramidal cell layer of rats. Neuroscience, 136, 757-767.

Sztainberg, Y., \& A. Chen (2010). An environmental enrichment model for mice. Nature Protocols, 5, 1535-1539.

van Praag, H., Kempermann, G., \& Gage, F.H. (2000). Neural consequences of environmental enrichment. Nature Reviews Neuroscience, 1(3), 191-198.

West, M.J. (2002). Design-based stereological methods for counting neurons. Progress in Brain Research, 135, 43-51.

West, M.J., Slomianka, L., \& Gundersen, H.J. (1991). Unbiased stereological estimation of the total number of neurons in the subdivisions of the rat hippocampus using the optical fractionator. Anatomical Record, 231, 482-497.

Wiesel, T.N., \& Hubel, D.H. (1963). Single-cell responses in striate cortex of kittens deprived of vision in one eye. Journal of Neurophysiology, 26, 1003-1017. 